\title{
On Stable Homotopy Types of Some Stunted Spaces
}

\author{
By \\ Hideaki ŌshimA*
}

\section{Introduction}

In this note we shall study the stable homotopy types ( $S$-types) of the stunted spaces $N_{k}^{n+k}(G)=N^{n+k}(G) / N^{k-1}(G)$, where $N^{n}(G)=S^{4 n+3}$ $\bmod G$ are quotients of $S^{4 n+3}$ by free orthogonal actions of a closed subgroup $G$ of $S^{3}$. In $\S 2$, we show that $N_{k}^{n+k}(G)$ are homeomorphic to the Thom spaces $N^{n}(G)^{k \xi}$. If $G$ is not finite, then $G$ is $S^{1}, S^{3}$ or the normalizer $N\left(S^{1}\right)$ of $S^{1}$ in $S^{3}$. The case with $G=S^{1}$ or $S^{3}$ has been treated by Feder and Gitler [8], [9]. We consider the case with $G$ $=N\left(S^{1}\right)$ in $\$ 3$. The case with $G=Z_{m}$ (cyclic group of order $m$ ) has been treated in [12], [15]. On and after $\S 4$, we consider the remaining cases, i.e. the cases with $G$ the binary dihedral or binary polyhedral groups (see $\$ 2$ for definitions). We examine the representation groups of the generalized quaternion groups $D^{*}\left(2^{m+1}\right)$ in $\S 4$ and evaluate the orders of some elements of $K_{F}\left(N^{n}\left(D^{*}\left(2^{m+1}\right)\right)\right)$ in $\S 5$ or $J\left(N^{n}\left(D^{*}\left(2^{m+1}\right)\right)\right)$ in $\S \S 6-7$ and study the $S$-types of $N_{k}^{n+k}(G)$ in the final section $\S 8$.

\section{Thom Spaces and Subgroups of $S^{3}$}

In this note $G$-space means a left $G$-space and $F$-vector space (or bundle) implies a right $F$-vector space (or bundle) for a field $F$. For a $G$-space $X$ its orbit space is denoted by $X \bmod G$ and if $G$ acts on $Y$ also, $X \times Y \bmod G$ denotes the orbit space by diagonal action. For a vector bundle $\alpha$ over a finite $C W$-complex $X, X^{\alpha}$ denotes the associated

Communicated by N. Shimada, February 5, 1975.

* Department of Mathematics, Osaka City University, Osaka, 
Thom space, that is, the one point compactification of the total space of $\alpha$.

Let $O_{F}(m)$ denote the orthogonal group $O(m)$ for $F=R$ (the real numbers), the unitary group $U(m)$ for $F=C$ (the complex numbers) and the symplectic group $S p(m)$ for $F=H$ (the quaternions) in $m$ dimensions respectively. We say that a representation $d: G \rightarrow O_{F}(m)$ of a topological group $G$ is free if the action of $G$ restricted to the unit sphere $S(V)$ is free, where $V$ is a representation space of $d$ with an inner product ( $\mid)$. Let $k V$ denote the sum $V \oplus \cdots \oplus V$ ( $k$ factors) with the inner product $(a \mid b)=\Sigma\left(a_{i} \mid b_{\imath}\right)$ for $a=\left(a_{1}, \ldots, a_{k}\right), b=\left(b_{1}, \ldots, b_{k}\right) \in k V$. For $k<k^{\prime}$, we regard $k V$ as a subspace of $k^{\prime} V$ by the identification $\left(a_{1}, \ldots, a_{k}\right)=\left(a_{1}\right.$, $\left.\ldots, a_{k}, 0, \ldots, 0\right)$.

For a given free representation $d: G \rightarrow O_{F}(m)$, we introduce the following notations:

$$
\begin{aligned}
N^{n}(G, d) & =S((n+1) V) \bmod G, \\
N_{k}^{n+k}(G, d) & =N^{n+k}(G, d) / N^{k-1}(G, d),
\end{aligned}
$$

and $\xi_{n}(G, d)$ means the canonical bundle

$$
S((n+1) V) \times V \bmod G \longrightarrow N^{n}(G, d)
$$

Then we have the following theorem.

Theorem 2.1. There exists a homeomorphism

$$
N_{k}^{n+k}(G, d) \approx N^{n}(G, d)^{k \xi_{n}(G, d)}
$$

Proof. Consider the map $f: S((n+1) V) \times D(k V) \rightarrow S((n+k+1) V)$ defined by

$$
f(x, y)=(y, x \lambda)
$$

where $D(k V)$ denotes the unit disk of $k V$ and $x=\left(x_{0}, \ldots, x_{n}\right) \in S((n+1) V)$, $y=\left(y_{0}, \ldots, y_{k-1}\right) \in D(k V), \lambda=\sqrt{1-(y \mid y)}$ and $x \lambda=\left(x_{0} \lambda, \ldots, x_{n} \lambda\right)$. It is easy to show that $f$ defines a $G$-equivariant homeomorphism

$$
S((n+1) V) \times(D(k V)-S(k V)) \longrightarrow S((n+k+1) V)-S(k V)
$$


and then we have a homeomorphism

$$
S((n+1) V) \times(D(k V)-S(k V)) \bmod G \longrightarrow(S((n+k+1) V)-S(k V)) \bmod G
$$

$S((n+1) V) \times(D(k V)-S(k V)) \bmod G$ may be identified with the total space of $k \xi_{n}(G, d)$. Compactifying the both spaces by adding one point, we have

$$
N^{n}(G, d)^{k \xi_{n}(G, d)} \approx N_{k}^{n+k}(G, d)
$$

as desired.

For example, we have

$$
S_{k}^{n+k}=S^{n+k} / S^{k-1} \approx S^{k} \vee S^{n+k}
$$

for the trivial representation $1 \rightarrow O(1)$ and

$$
F P_{k}^{n+k}=F P^{n+k} / F P^{k-1} \approx\left(F P^{n}\right)^{k \xi}
$$

for the identity $O_{F}(1) \rightarrow O_{F}(1)$ and

$$
L_{k}^{n+h}(m)=L^{n+h}(m) / L^{k-1}(m) \approx L^{n}(m)^{k \xi}
$$

for $Z_{m} \subset U(1)$, where $F P^{n}$ indicatcs the $F$-projective space and $L^{n}(m)$ the standard $\bmod m$ lens space. These are well-known.

We say that two spaces $X$ and $Y$ are stably homotopy equivalent ( $S$-equivalent) if the suspensions $S^{u} \wedge X$ and $S^{v} \wedge Y$ are homotopy equivalent for some $u$ and $v$.

The classifications of $S$-types of $S_{k}^{n+k}, C P_{k}^{n+k}$ and $H P_{k}^{n+k}$ have been completed. The sphere case is trivial and the complex or quaternion projective space case has been done by Feder and Gitler [8], [9].

It is known that which compact group admit a free representation. Finite groups admitting a free representation are listed in [23, Chapter 6]. If a compact group $G$ including infinite elements has a free representation, then $G$ is a Lie group ([17, V Th. 2]) and must be $S^{1}, S^{3}$ or the normalizer $N\left(S^{1}\right)$ of $S^{1}$ in $S^{3}$ [5, III 8.5].

From now on, we will treat the case with $G$ a closed subgroup of $S^{3}$ and $d$ the inclusion $d_{1}: G \subset S^{3}=S p(1)$. And we will use the notations: 


$$
\begin{aligned}
& N^{n}(G)=N^{n}\left(G, d_{1}\right), \\
& N_{k}^{n+k}(G)=N_{k}^{n+k}\left(G, d_{1}\right), \\
& \xi_{n}(G)=\xi_{n}\left(G, d_{1}\right),
\end{aligned}
$$

$\xi_{n}(G)_{F}$, the underlying $F$-vector bundle of $\xi_{n}(G)$.

$\pi(G, H): N^{n}(G) \rightarrow N^{n}(H)$, the natural projection for $G \subset H \subset S^{3}$. Closed subgroups of $S^{3}$ are maximal tori $S^{1}, N\left(S^{1}\right)^{\text {'s }}$ (any two of them are conjugate each other respectively), $S^{3}$ itself or finite subgroups. Concerning finite subgroups of $S^{3}$, we have

2.2 (Wolf [23, 2.6.7]). Every finite subgroup of $S^{3}$ is a cyclic, binary dihedral or binary polyhedral group. If two finite subgroups of $S^{3}$ are isomorphic, they are conjugate in $S^{3}$.

We remark that if two subgroups $G, H$ of $S^{3}$ are conjugate, then $N^{n}(G)$ and $N^{n}(H)$ are naturally homeomorphic, and this homeomorphism induces the isomorphism between $\xi_{n}(G)$ and $\xi_{n}(H)$. Thus we may assume that $N^{n}(G)$ and $\xi_{n}(G)$ are defined for the conjugate classes of subgroups of $S^{3}$. So we describe the subgroups of $S^{3}$ in terms of generators and relations as follows: the binary dihedral group $D^{*}(4 m)$ of order $4 m$ $(m \geqq 2)$, the binary tetrahedral group $T^{*}$ of order 24 , the binary octahedral group $O^{*}$ of order 48 and the binary icosahedral group $I^{*}$ of order 120 are given by

$$
\begin{aligned}
& D^{*}(4 m): x^{m}=(y x)^{2}=y^{2}, \\
& T^{*} \quad: x^{3}=(y x)^{3}=y^{2}, y^{4}=1, \\
& O^{*} \quad: x^{4}=(y x)^{3}=y^{2}, y^{4}=1, \\
& I^{*} \quad: x^{5}=(y x)^{3}=y^{2}, y^{4}=1
\end{aligned}
$$

(see [23] or $[18,6.2]$ ). $T^{*}, O^{*}$ and $I^{*}$ are called the binary polyhedral groups. $D^{*}\left(2^{m+1}\right)$ is called the generalized quaternion group. 


\section{3. $\mathbb{N}^{n}\left(\mathbb{N}\left(S^{1}\right)\right)$}

In this section we examine the $S$-types of $N_{k}^{n+k}\left(N\left(S^{1}\right)\right)$.

For simplicity we use the notations

$$
N^{n}=N^{n}\left(N\left(S^{1}\right)\right), N_{k}^{n+k}=N_{k}^{n+k}\left(N\left(S^{1}\right)\right) .
$$

For $0 \leqq k \leqq n$, we define the cells in $S((n+1) H)=S^{4 n+3}$ as follows:

$$
\begin{gathered}
\underline{e}^{4 k}=\left\{\left(z_{1}, \ldots, z_{2 k+1}, 0, \ldots, 0\right) ; z_{2 k+1} \neq 0, \arg \left(z_{2 k+1}\right)=0\right\}, \\
\underline{e}^{4 k+1}=\left\{\left(z_{1}, \ldots, z_{2 k+2}, 0, \ldots, 0\right) ; z_{2 k+1} \neq 0, z_{2 k+2} \neq 0,\right. \\
\left.\arg \left(z_{2 k+1}\right)=\arg \left(z_{2 k+2}\right)=0\right\}, \\
\underline{e}^{4 k+2}=\left\{\left(z_{1}, \ldots, z_{2 k+2}, 0, \ldots, 0\right) ; z_{2 k+1} \neq 0,0<\arg \left(z_{2 k+1}\right)<\pi,\right. \\
\left.z_{2 k+2} \neq 0, \arg \left(z_{2 k+2}\right)=0\right\},
\end{gathered}
$$

and their images in $N^{n}$ by the natural projection $S^{4 n+3} \rightarrow N^{n}$ are denoted by $e^{4 k}, e^{4 k+1}$ and $e^{4 k+2}$ respectively, here we regard $H$ as the complex 2 -space by the replacement $q=z+z^{\prime} j$. Then it is easy to check the following proposition.

Proposition 3.1. $\left\{e^{4 k}, e^{4 k+1}, e^{4 k+2} ; 0 \leqq k \leqq n\right\}$ gives a $C W$-decomposition of $N^{n}$.

Remark that the above $C W$-decomposition satisfies the condition that the $4 m+2$-skeleton of $N^{n}$ is $N^{m}$ for $0 \leqq m \leqq n$.

It is easy to show that the Serre spectral sequence of the fibration

$$
N^{0}=R P^{2} \longrightarrow N^{n} \longrightarrow H P^{n}
$$

is trivial and therefore we have the following proposition.

Proposition 3.2. For any coefficients A, we have

$$
H^{*}\left(N^{n} ; A\right) \cong H^{*}\left(H P^{n} ; \mathbb{Z}\right) \otimes H^{*}\left(R P^{2} ; A\right) .
$$

Let $K_{F}$ be $\operatorname{real}(F=\mathbf{R})$, complex $(F=\mathbb{C})$ or $\operatorname{symplectic}(F=\mathbf{H}) K$-theory 
and $\theta_{F}$ be the representation

$$
N\left(S^{1}\right) \underset{\text { quotient }}{\longrightarrow} N\left(S^{1}\right) / S^{1}=O(1) \subset O_{F}(1)
$$

and $\hat{\theta}_{F}$ be the associated $F$-line bundle

$$
S((n+1) H) \times F \bmod N\left(S^{1}\right) \longrightarrow N^{n}
$$

Proposition 3.3. There exists a split exact sequence

$$
0 \longrightarrow K_{F}\left(H P^{n}\right) \longrightarrow K_{F}\left(N^{n}\right) \longrightarrow Z_{2^{f(n ; F)}} \longrightarrow 0
$$

where $f(n ; \mathbb{R})=2[n / 2]+2, f(n ; \mathbb{C})=n+1, f(n ; \mathbb{H})=2[(n+1) / 2]$ and the reduced element $\hat{\theta}_{F}-1 \in \widetilde{K}_{F}\left(N^{n}\right)$ generates the direct summand $Z_{2 f(n ; F)}$. Here [a] denotes the greatest integer which does not exceed $a$.

Proof. (i) $F=\mathrm{C}$-case. Consider the commutative triangle

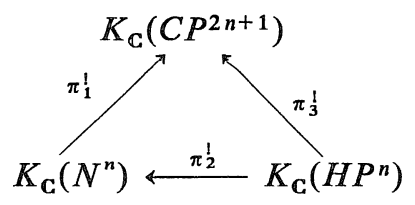

where $\pi_{1}=\pi\left(S^{1}, N\left(S^{1}\right)\right), \pi_{2}=\pi\left(N\left(S^{1}\right), S^{3}\right)$ and $\pi_{3}=\pi\left(S^{1}, S^{3}\right)$. Let $\eta_{2 n+1}$ be the canonical complex line bundle over $C P^{2 n+1}$. Put $\mu=\eta_{2 n+1}-1$ $\in \tilde{K}_{\mathbf{C}}\left(C P^{2 n+1}\right)$ and $v_{n}=\xi_{n}\left(S^{3}\right)_{\mathbf{C}}-2 \in \tilde{K}_{\mathbf{C}}\left(H P^{n}\right)$. Then it is well-known that

$$
\begin{aligned}
& K_{\mathbf{C}}\left(C P^{2 n+1}\right)=\mathbb{Z}[\mu] / \mu^{2 n+2}, \\
& K_{\mathbf{C}}\left(H P^{n}\right)=\mathbb{Z}\left[v_{n}\right] / v_{n}^{n+1}, \\
& \pi_{3}^{*}\left(\xi_{n}\left(S^{3}\right)_{\mathbb{C}}\right)=\eta_{2 n+1} \oplus \bar{\eta}_{2 n+1}
\end{aligned}
$$

where ${ }^{-}$denotes the complex conjugation. Since

$$
\pi_{3}^{1}\left(v_{n}\right)=\mu+\bar{\mu}=\mu^{2}-\mu^{3}+\cdots-\mu^{2 n+1},
$$

the image $\operatorname{Im} \pi_{3}^{1}$ of $\pi_{3}^{1}$ is a direct summand of $K_{\mathbf{C}}\left(C P^{2 n+1}\right)$. In the commutative diagram 


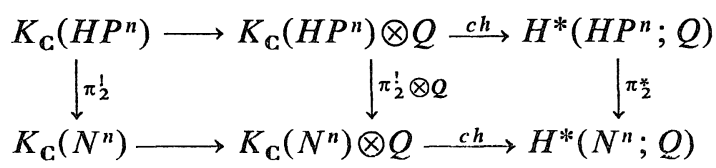

$\pi_{2}^{*}$ is an isomorphism by (3.2) and then $\pi_{2}^{\prime}$ is monomorphic and the cokernel of $\pi_{2}^{1}$ is finite, where ch denotes the Chern character. And therefore $I m \pi_{3}^{!}$and $I m \pi_{1}^{!}$have the same rank. Then, since $\operatorname{Im} \pi_{3}^{1} \subset \operatorname{Im} \pi_{1}^{!}$ and $\operatorname{Im} \pi_{3}^{1}$ is a direct summand of the free module $K_{\mathrm{C}}\left(C P^{2 n+1}\right)$, we know that $\operatorname{Im} \pi_{3}^{1}=\operatorname{Im} \pi_{1}^{1}$ and therefore $\pi_{2}^{1}$ is an isomorphism onto a direct summand of $K_{\mathbf{C}}\left(N^{n}\right)$.

By definition, we have

$$
\pi_{2}^{!}\left(\hat{\theta}_{\mathbf{c}}-1\right)=0
$$

and then the aboves imply that $\hat{\theta}_{\mathbb{C}}-1$ has a finite order. Put $\sigma=\pi\left(\mathbb{Z}_{4}\right.$, $\left.S^{1}\right)^{!} \mu \in K_{\mathbf{C}}\left(L^{2 n+1}(4)\right)$. It is easy to see that

$$
\pi\left(\mathbf{Z}_{4}, N\left(S^{1}\right)\right)^{\prime}\left(\hat{\theta}_{\mathbf{C}}-1\right)=\sigma^{2}+2 \sigma .
$$

Since the order $\#\left(\sigma^{2}+2 \sigma\right)$ of $\sigma^{2}+2 \sigma$ is $2^{n+1}\left[14\right.$, Th. A], $\#\left(\hat{\theta}_{\mathbf{C}}-1\right)$ is a multiple of $2^{n+1}$.

Let $\left\{E_{r}\right\}$ be the Atiyah-Hirzebruch spectral sequence for $K_{\mathbf{C}}^{*}\left(N^{n}\right)$. Then $E_{2}^{p, q}=H^{p}\left(N^{n} ; K_{\mathbf{C}}^{q}\right)$ and

$$
\# \operatorname{Tor}\left(K_{\mathbf{C}}\left(N^{n}\right)\right) \leqq \# \operatorname{Tor}\left(\sum_{p} E_{2}^{p,-p}\right)=\# \sum_{k=0}^{n} E_{2}^{4 k+2,-4 k-2}=2^{n+1}
$$

by (3.2), where Tor $(A)$ denotes the torsion submodule of a module $A$. Hence $\#\left(\hat{\theta}_{\mathbf{C}}-1\right)$ is a divisor of $2^{n+1}$. Therefore $\#\left(\hat{\theta}_{\mathbf{C}}-1\right)=2^{n+1}$ and $\hat{\theta}_{\mathbf{C}}$ -1 generates $\operatorname{Tor}\left(K_{\mathbf{C}}\left(N^{n}\right)\right)$. This completes the proof of the proposition for $F=\mathbb{C}$.

Remark. (3.2) implies that the above spectral sequence collapses.

(ii) $F=\mathbf{R}$-case. Let $c: K_{\mathbf{R}} \rightarrow K_{\mathbf{C}}$ be the complexification and $r: K_{\mathbf{C}}$ $\rightarrow K_{\mathbf{R}}$ be the real restriction. Since $r_{\circ} c=2$ and $c\left(\hat{\theta}_{\mathbf{R}}-1\right)=\hat{\theta}_{\mathbf{C}}-1$, we have

$$
\#\left(\hat{\theta}_{\mathbf{R}}-1\right)=2^{n+1} \quad \text { or } \quad 2^{n+2}
$$

by (i).

Consider the Atiyah-Hirzebruch spectral sequence $\left\{_{\mathbb{R}} E_{\boldsymbol{r}}\right\}$ for $K_{\mathbf{R}}^{*}\left(N^{n}\right)$. 
Then ${ }_{\mathbf{R}} E_{2}^{p, q}=H^{p}\left(N^{n} ; K_{\mathbf{R}}^{q}\right)$ and

$$
\text { \# } \operatorname{Tor}\left(K_{\mathbf{R}}\left(N^{n}\right)\right) \leqq \# \operatorname{Tor}\left(\sum_{\boldsymbol{p}} E_{\infty}^{p,-p}\right) .
$$

Since the rank of $K_{\mathbf{R}}\left(N^{n}\right)$ equals the rank of $K_{\mathbf{C}}\left(N^{n}\right), n+1$, we have

$$
{ }_{\mathbf{R}} E_{\infty}^{4 k,-4 k} \cong Z \quad \text { for } \quad 0 \leqq k \leqq n,
$$

and then

$$
\# \operatorname{Tor}\left(\sum_{p} E_{\infty}^{p,-p}\right)=\# \operatorname{Tor}\left(\sum_{p \equiv 1,2(8)} E_{\infty}^{p,-p}\right) \leqq \# \sum_{p \equiv 1,2(8)}{ }_{\mathbf{R}} E_{2}^{p,-p}=2^{2\left[\frac{n+2}{2}\right]} .
$$

Then we have

$$
\# \operatorname{Tor}\left(K_{\mathbf{R}}\left(N^{n}\right)\right) \leqq 2^{2[(n+2) / 2]} \text {. }
$$

Since

$$
f(n ; \mathbf{R})=2[(n+2) / 2]= \begin{cases}n+1 & \text { if } n \text { is odd } \\ n+2 & \text { if } n \text { is even }\end{cases}
$$

we know that

$$
\#\left(\hat{\theta}_{\mathbf{R}}-1\right)=2^{n+1} \quad \text { for } n \text { odd }
$$

An easy computation shows that

$$
H^{*}\left(N^{2 m+1}, N^{2 m} ; A\right) \cong H^{*}\left(H P^{2 m+1}, H P^{2 m} ; \mathbb{Z}\right) \otimes H^{*}\left(R P^{2} ; A\right)
$$

and then by the Atiyah-Hirzebruch spectral sequence, we have

$$
K_{\mathbf{R}}^{ \pm 1}\left(N^{2 m+1}, N^{2 m}\right)=0, \quad K_{\mathbf{R}}\left(N^{2 m+1}, N^{2 m}\right) \cong Z .
$$

Then the long exact sequence of the pair $\left(N^{2 m+1}, N^{2 m}\right)$ induces the following short exact sequence

$$
0 \longrightarrow K_{\mathbf{R}}\left(N^{2 m+1}, N^{2 m}\right) \longrightarrow K_{\mathbf{R}}\left(N^{2 m+1}\right) \stackrel{\iota^{!}}{\longrightarrow} K_{\mathbf{R}}\left(N^{2 m}\right) \longrightarrow 0 .
$$

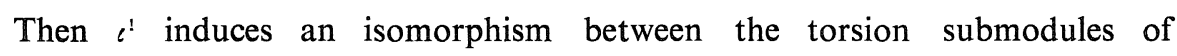
$K_{\mathbf{R}}\left(N^{2 m+1}\right)$ and $K_{\mathbf{R}}\left(N^{2 m}\right)$. And since $\iota^{\prime}\left(\hat{\theta}_{\mathbf{R}}-1\right)=\hat{\theta}_{\mathbf{R}}-1$, we have

$$
\#\left(\hat{\theta}_{\mathbf{R}}-1\right)=2^{n+2} \quad \text { for } n \text { even. }
$$


Therefore

$$
\#\left(\hat{\theta}_{\mathbf{R}}-1\right)=2^{f(n ; \mathbf{R})}
$$

and $\hat{\theta}_{\mathbf{R}}-1$ generates $\operatorname{Tor}\left(K_{\mathbf{R}}\left(N^{n}\right)\right)$.

Let $N_{(s)}^{n}$ be the $s$-skeleton of $N^{n}$ with respect to the $C W$-decomposition (3.1) and $K_{\mathbf{R}}\left(N^{n}\right)_{s}$ be the kernel of the restriction $K_{\mathbf{R}}\left(N^{n}\right) \rightarrow K_{\mathbf{R}}\left(N_{(s-1)}^{n}\right)$. Then ${ }_{\mathbf{R}} E_{\infty}^{s,-s}=K_{\mathbf{R}}\left(N^{n}\right)_{s} / K_{\mathbf{R}}\left(N^{n}\right)_{s+1}$. The facts $\operatorname{Tor}\left(K_{\mathbf{R}}\left(N^{n}\right)\right)=Z_{2^{f(n ; \mathbf{R})}}$ and \# $\operatorname{Tor}\left(\sum_{p} E_{2}^{p,-p}\right)=2^{f(n ; \mathbf{R})}$ imply that we may think that ${ }_{\mathbf{R}} E_{\infty}^{4 k,-4 k} \cong Z(0 \leqq$ $k \leqq n)$ is a direct summand of $K_{\mathbf{R}}\left(N^{n}\right)$, that is, an element of $K_{\mathbf{R}}\left(N^{n}\right)_{4 k}$ which represents a generator of ${ }_{\mathbf{R}} E_{\infty}^{4 k,-4 k}=Z$ generates a direct summand of $K_{\mathbf{R}}\left(N^{n}\right)$.

Put $\underline{v}=\pi_{2}^{1}\left(v_{n}\right) \in K_{\mathbf{C}}\left(N^{n}\right)$. Comparing the spectral sequences for $K_{\mathbf{C}}\left(H P^{n}\right)$ and $K_{\mathbf{C}}\left(N^{n}\right)$, we know that $\underline{v}^{s}$ represents a generator of $E_{\infty}^{4 s,-4 s}$ $=E_{2}^{4 s,-4 s}$.

Let $c:{ }_{\mathbf{R}} E_{r} \rightarrow E_{r}$ be the homomorphism induced by the complexification $c: K_{\mathbf{R}}\left(N^{n}\right) \rightarrow K_{\mathbf{C}}\left(N^{n}\right)$. Since $c:{ }_{\mathbf{R}} E_{2}^{p, q}=H^{p}\left(N^{n} ; K_{\mathbf{R}}^{q}\right) \rightarrow E_{2}^{p, q}=H^{p}\left(N^{n} ; K_{\mathbf{C}}^{q}\right)$ is induced by the coefficients homomorphism $c: K_{\mathrm{R}}^{q} \rightarrow K_{\mathrm{C}}^{q}, c:{ }_{\mathrm{R}} E_{2}^{8 k+4,-8 k-4}$ $\rightarrow E_{2}^{8 k+4,-8 k-4}$ coincides with the multiplication $H^{8 k+4}\left(N^{n} ; \mathbb{Z}\right) \rightarrow H^{8 k+4}\left(N^{n}\right.$; $\mathbb{Z})$ by 2 . Then the aboves imply that $\underline{v}^{s}$ is not in the image of $c$ : $K_{\mathbf{R}}\left(N^{n}\right) \rightarrow K_{\mathbf{C}}\left(N^{n}\right)$ for $s$ odd and $0 \leqq s \leqq n$. On the other hand, the image of $c: K_{\mathbf{R}}\left(H P^{n}\right) \rightarrow K_{\mathbf{C}}\left(H P^{n}\right)$ is generated by $\varepsilon_{k} v^{k}$ for $0 \leqq k \leqq n$, where $\varepsilon_{k}=1$ for $k$ odd or 2 for $k$ even $[20,3.11]$. Then (i) and the commutative diagram

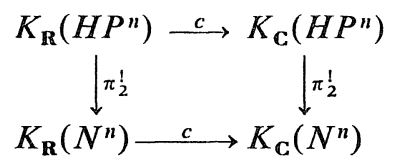

imply that the composition

$$
K_{\mathbf{R}}\left(H P^{n}\right) \stackrel{\pi_{2}^{1}}{\longrightarrow} K_{\mathbf{R}}\left(N^{n}\right) \longrightarrow K_{\mathbf{R}}\left(N^{n}\right) / \text { Tor }
$$

is an isomorphism. Therefore we have the split exact sequence

$$
0 \longrightarrow K_{\mathbf{R}}\left(H P^{n}\right) \longrightarrow K_{\mathbf{R}}\left(N^{n}\right) \longrightarrow Z_{2 f(n ; \mathbf{R})} \longrightarrow 0
$$

as desired. 
(iii) $F=\mathbf{H}$-case. Identifying $K S p$ with $K_{\mathbf{R}}^{-4}$, we can prove the proposition for $F=\mathbf{H}$ by the same methods with (ii). And we complete the proof of Proposition.

Corollary 3.4. (i) We have the exact sequence

$$
0 \longrightarrow \tilde{K}_{F}\left(N_{k}^{n+k}\right) \longrightarrow K_{F}\left(N^{n+k}\right) \longrightarrow K_{F}\left(N^{k-1}\right) \longrightarrow 0 .
$$

(ii) The complex conjugation $t: K_{\mathbb{C}}\left(N^{n}\right) \longrightarrow K_{\mathbb{C}}\left(N^{n}\right)$ is the identity.

Proof. (3.3) and the exact sequence

$$
0 \longrightarrow \widetilde{K}_{F}\left(H P_{k}^{n+k}\right) \longrightarrow K_{F}\left(H P^{n+k}\right) \longrightarrow K_{F}\left(H P^{k-1}\right) \longrightarrow 0
$$

imply (i). Since

$$
t\left(\hat{\theta}_{\mathbf{C}}\right)=\operatorname{toc}\left(\hat{\theta}_{\mathbf{R}}\right)=c\left(\hat{\theta}_{\mathbf{R}}\right)=\hat{\theta}_{\mathbf{C}}
$$

and $t: K_{\mathbf{C}}\left(H P^{n}\right) \rightarrow K_{\mathbf{C}}\left(H P^{n}\right)$ is the identity function, we have (ii) by (3.3).

We shall evaluate the $J$-groups $J\left(N^{n}\right)$ [3]. Let $\Psi_{F}^{k}: K_{F}(X) \rightarrow K_{F}(X)$ be the Adams operation for $F=\mathbb{R}$ or $\mathbf{C}$. By now proved Adams conjecture [2] we may identify $J(X)$ with $\tilde{K}_{\mathbf{R}}(X) / \bigcap_{e} Y_{e}$, where $e: \mathbb{Z} \rightarrow\{0,1$, $2, \ldots\}$ and $Y_{e}=\sum_{k \in \mathbf{Z}} k^{e(k)}\left(\Psi_{\mathbf{R}}^{k}-1\right) K_{\mathbf{R}}(X)$. We have

$$
\Psi_{R}^{k}\left(\hat{\theta}_{\mathbf{R}}\right)=\hat{\theta}_{\mathbb{R}}^{k}= \begin{cases}\hat{\theta}_{\mathbf{R}} & \text { if } k \text { is odd } \\ 1 & \text { if } k \text { is even }\end{cases}
$$

Then, since $\Psi_{\mathbb{R}}^{k}$ commutes with $\pi_{2}^{1}$, we have the following proposition by (3.3).

Proposition 3.5. There exists a split exact sequence

$$
0 \longrightarrow J\left(H P^{n}\right) \longrightarrow J\left(N^{n}\right) \longrightarrow Z_{2^{2[n / 2]+2}} \longrightarrow 0
$$

and then the J-orders of the canonical symplectic line bundles over $H P^{n}$ and $N^{n}$ are equal.

Let $B_{n}$ be the $J$-order of the canonical symplectic line bundle $\xi_{n}\left(S^{3}\right)$ over $H P^{n}$. $\left(B_{n}\right.$ has been computed by Sigrist and Suter [21].) Then 
by Atiyah $[3,2.6]$ we have

Theorem 3.6. If $k-l \equiv 0\left(B_{n}\right)$, then $N_{k}^{n+k}$ and $N_{l}^{n+l}$ are of the same stable homotopy type.

Using above propositions and corollary, we may prove the following theorem by following faithfully the proof of $[9, \S 4]$ which has treated $H P_{k}^{n+k}$ instead of $N_{k}^{n+k}$.

Theorem 3.7. If $N_{k}^{n+k}$ and $N_{l}^{m+l}$ are of the same stable homotopy type, then $m=n$ and one of the following conditions hold:

$$
\begin{aligned}
& \text { (i) } k-l \equiv 0\left(B_{n}\right) \\
& \text { (ii) } k-l \equiv 0\left(B_{n-l}\right) \text { and } k+l \equiv 0\left(B_{n}\right) \text {. }
\end{aligned}
$$

\section{Representations of the Generalized Quaternion Groups}

In this section we examine the representation groups of the generalized quaternion groups $D^{*}\left(2^{m+1}\right)$ according to Pitt [19].

Let $R_{F}(G)$ denote $\operatorname{real}(F=\mathbb{R})$, complex $(F=\mathbb{C})$ or $\operatorname{symplectic}(F=\mathbf{H})$ representation group of a group $G$. There exist the natural homomorphisms

$$
R_{\mathbf{R}}(G) \underset{r}{\stackrel{c_{\mathbf{R}}}{\rightleftarrows}} R_{\mathbf{C}}(G) \stackrel{h}{\stackrel{h}{\rightleftarrows}} R_{\mathbf{H}}(G)
$$

satisfying the relations

$$
\begin{array}{ll}
r \circ c_{\mathbb{R}}=2, & c_{\mathbb{R}^{\circ}}=1+t \\
h \circ c^{\prime}=2, & c^{\prime} \circ h=1+t,
\end{array}
$$

where $t: R_{\mathbf{C}}(G) \rightarrow R_{\mathbf{C}}(G)$ is complex conjugation. Being $R_{F}(G)$ free, $c_{\mathbb{R}}$ and $c^{\prime}$ are monomorphisms and in what follows we shall identify $R_{\mathbf{R}}(G)$ and $R_{H}(G)$ with their images in $R_{\mathbf{C}}(G)$ under $c_{\mathbf{R}}$ and $c^{\prime}$.

Recall that $D^{*}\left(2^{m+1}\right)=\left\{x, y ; x^{2^{m-1}}=(y x)^{2}=y^{2}\right\}$. We consider the following complex representations of $D^{*}\left(2^{m+1}\right)$ : 


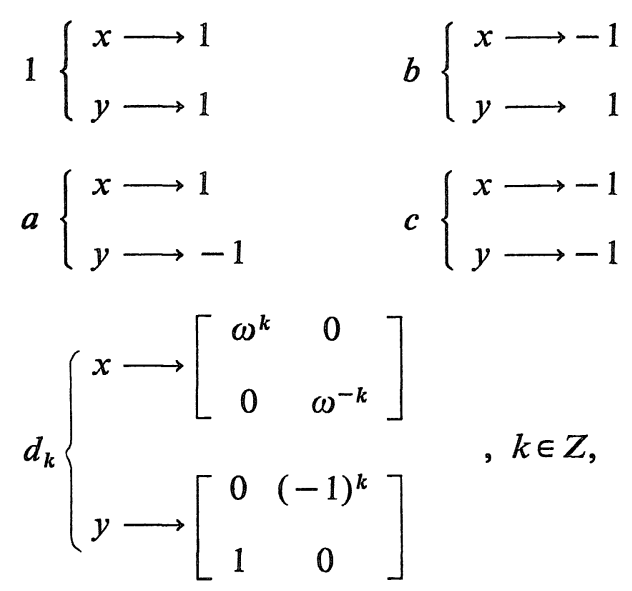

where $\omega$ is a primitive $2^{m}$-th root of unity. The characters of these representations are

$$
\begin{array}{ll}
\chi_{1}\left(x^{u} y^{v}\right)=1, & \chi_{b}\left(x^{u} y^{v}\right)=(-1)^{u}, \\
\chi_{a}\left(x^{u} y^{v}\right)=(-1)^{v}, & \chi_{c}\left(x^{u} y^{v}\right)=(-1)^{u+v}, \\
\chi_{d_{k}}\left(x^{u} y^{v}\right)=\left(\omega^{u k}+\omega^{-u k}\right)(1-v),
\end{array}
$$

where $u=1,2, \ldots, 2^{m}, v=0,1$. Evaluating the characters, we have the relations

$$
a^{2}=b^{2}=c^{2}=1, \quad a b=c, \quad b c=a, \quad c a=b,
$$

4.1. $d_{0}=1+a$,

$$
d_{2^{m-1}}=b+c, \quad d_{-k}=d_{k},
$$$$
d_{2^{m-1+k}}=d_{2^{m-1-k}}, \quad d_{k} d_{j}=d_{k+j}+d_{k-j}, \quad a d_{k}=d_{k},
$$$$
b d_{k}=c d_{k}=d_{2^{m-1-k}} \text {. }
$$

Then we have

4.2. $R_{\mathbf{C}}\left(D^{*}\left(2^{m+1}\right)\right)$ is free abelian on $1, b$ and $d_{k}\left(0 \leqq k \leqq 2^{m-1}\right)$ and generated multiplicatively by $1, a, b$ and $d_{1}$. Therefore $t=i d e n t i t y$ on $R_{\mathrm{C}}\left(D^{*}\left(2^{m+1}\right)\right)$.

4.3. $R_{\mathbf{R}}\left(D^{*}\left(2^{m+1}\right)\right)$ is free abelian on $1, b, d_{2 k}\left(0 \leqq k \leqq 2^{m-2}\right)$ and $2 d_{2 k+1}$ $\left(0 \leqq k<2^{m-2}\right)$ and generated multiplicatively by $1, a, b, 2 d_{1}$ and $d_{1}^{2}$. 
4.4. $R_{\mathbf{H}}\left(D^{*}\left(2^{m+1}\right)\right)$ is free abelian on $2,2 b, 2 d_{2 k}\left(0 \leqq k \leqq 2^{m-2}\right)$ and $d_{2 k+1}\left(0 \leqq k<2^{m-2}\right)$.

Let $\lambda^{k}(\quad)$ be the exterior $k$-th power operation and put

$$
\lambda_{t}(x)=\sum_{k \geqq 0} \lambda^{k}(x) t^{k} \in R_{\mathbf{C}}(G)[[t]] \quad \text { for } \quad x \in R_{\mathbf{C}}(G) .
$$

Then it is well known that

$$
\lambda_{t}(x+y)=\lambda_{t}(x) \lambda_{t}(y)
$$

Hence

$$
\lambda_{t}\left(n d_{1}\right)=\left(\lambda_{t}\left(d_{1}\right)\right)^{n}=\left(1+d_{1} t+t^{2}\right)^{n}
$$

Therefore we have

$$
\text { Lemma 4.5. } \quad \lambda_{-1}\left(n d_{1}\right)=\left(2-d_{1}\right)^{n} \text {. }
$$

For the proof of Proposition 5.7, we prepare the following lemma.

Lemma 4.6. In $R_{\mathbf{C}}\left(D^{*}\left(2^{m+1}\right)\right)$ we have the relations

$$
\begin{aligned}
& d_{1}^{2 k}=\frac{1}{2} \sum_{-\infty<t<\infty}\left(\begin{array}{c}
2 k \\
k+2^{m-1} t
\end{array}\right) d_{0}+\sum_{j=1}^{2^{m-2}-1} \sum_{-\infty<t<\infty}\left(\begin{array}{c}
2 k \\
k+2^{m-1} t-j
\end{array}\right) d_{2 j} \\
& +\frac{1}{2} \sum_{-\infty<t<\infty}\left(\begin{array}{c}
2 k \\
k+2^{m-1} t-2^{m-2}
\end{array}\right) d_{2^{m-1}} \\
& d_{1}^{2 k+1}=\sum_{j=0}^{2^{m-2}-1} \sum_{-\infty<t<\infty}\left(\begin{array}{c}
2 k+1 \\
k+2^{m-1} t-j
\end{array}\right) d_{2 j+1} .
\end{aligned}
$$

Proof. Using (4.1), we may prove this by induction on $k$. The proof is elementary and easy, so we omit it.

\section{5. $\boldsymbol{K}_{\boldsymbol{F}}\left(\mathbb{N}^{n}(\boldsymbol{G})\right)$}

Hereafter $G$ denotes a finite subgroup of $S^{3}$.

Let $V(=H)$ be the representation space of $d_{1}: G \subset S^{3}=S p(1)=S U(2)$. Put $E=(n+1) V$ and consider the following exact sequence of equivariant K-theory. 


$$
\begin{aligned}
& \cdots \longrightarrow K F_{G}(D E, S E) \longrightarrow K F_{G}(D E) \longrightarrow \\
& K F_{G}(S E) \longrightarrow K F_{G}^{1}(D E, S E) \longrightarrow \cdots
\end{aligned}
$$

where $F$ denotes $\mathbb{R}$ or $\mathbb{C}$. By Thom isomorphism, this induces the exact sequence

$$
\begin{aligned}
\cdots \longrightarrow K F_{G}^{-4 n-4}(p t .) \stackrel{\psi_{F}}{\longrightarrow} & R_{F}(G) \stackrel{\phi_{F}}{\longrightarrow} K_{F}\left(N^{n}(G)\right) \\
& \longrightarrow K F_{G}^{1-4 n-4}(p t .) \longrightarrow \cdots
\end{aligned}
$$

Recalling that

$$
\begin{aligned}
& K C_{G}^{-4 n-4}(p t .)=R_{C}(G), \\
& K R_{G}^{-4 n-4}(p t .)= \begin{cases}R_{R}(G) & \text { if } n \text { is odd } \\
R_{H}(G) & \text { if } n \text { is even, }\end{cases}
\end{aligned}
$$

then $\psi_{F}$ is the multiplication by $\lambda_{-1}\left((n+1) d_{1}\right)=\left(2-d_{1}\right)^{n+1}$ which is contained in $R_{\mathbf{R}}(G)$ (if $n$ is odd) or $R_{\mathbb{H}}(G)$ (if $n$ is even). When $F=\mathbb{C}$, these are as usual. In case $F=R$, see [19]. $\phi_{F}$ maps a representation of $G$ to its associated vector bundle induced from the principal $G$ bundle $S((n+1) V) \rightarrow N^{n}(G)$. Hence $\phi_{\mathbf{C}}\left(d_{1}\right)=\xi_{n}(G)_{\mathbb{C}}$ and $\phi_{\mathbf{R}}\left(r\left(d_{1}\right)\right)=\xi_{n}(G)_{\mathbf{R}}$. Since $K C_{G}^{\text {odd }}(p t)=$.0 and $K R_{G}^{-k}(p t)=$.0 for $k \equiv 3,7(8)([4])$, we obtain the cxact sequence

$$
K F_{G}^{-4 n-4}(p t .) \stackrel{\psi_{F}}{\longrightarrow} R_{F}(G) \stackrel{\phi_{F}}{\longrightarrow} K_{F}\left(N^{n}(G)\right) \longrightarrow 0,
$$

and then we have

Proposition 5.1. (cf. [10], [19]) $\phi_{F}: R_{F}(G) \rightarrow K_{F}\left(N^{n}(G)\right.$ ) induces the isomorphisms

$$
\begin{aligned}
& K_{\mathbf{C}}\left(N^{n}(G)\right) \cong R_{\mathbf{C}}(G) /\left(2-d_{1}\right)^{n+1} R_{\mathbf{G}}(G) \\
& K_{\mathbf{R}}\left(N^{n}(G)\right) \cong\left\{\begin{array}{l}
R_{\mathbf{R}}(G) /\left(2-d_{1}\right)^{n+1} R_{\mathbf{R}}(G) \quad \text { if } n \text { is odd } \\
R_{\mathbf{R}}(G) /\left(2-d_{1}\right)^{n+1} R_{\mathbf{H}}(G) \quad \text { if } n \text { is even. }
\end{array}\right.
\end{aligned}
$$

In the rest of this section, we consider the case with $G$ a generalized quaternion group $D^{*}\left(2^{m+1}\right)$. For simplicity we will use the nota- 
tions

$$
\begin{aligned}
& N^{n}(m)=N^{n}\left(D^{*}\left(2^{m+1}\right)\right), \quad N_{k}^{n+k}(m)=N^{n+k}(m) / N^{k-1}(m), \\
& \xi_{n}(m)=\xi_{n}\left(D^{*}\left(2^{m+1}\right)\right), \\
& \delta_{n}^{\prime}(m)=\xi_{n}(m)_{\mathbf{C}}-2 \in \tilde{K}_{\mathbf{C}}\left(N^{n}(m)\right) \text { and } \\
& \delta_{n}(m)=\xi_{n}(m)_{\mathbf{R}}-4 \in \widetilde{K}_{\mathbf{R}}\left(N^{n}(m)\right) .
\end{aligned}
$$

The remaining part of this section is devoted to evaluate the orders of $\delta_{n}^{\prime}(m)=\phi_{\mathbf{C}}\left(d_{1}-2\right)$ and $\delta_{n}(m)=\phi_{\mathbf{R}}\left(r\left(d_{1}\right)-4\right)$.

\section{Proposition 5.2.}

$$
\# \delta_{n}^{\prime}(m)^{k}= \begin{cases}2^{m+2(n-k)+1} & \text { if } 1 \leqq k \leqq n \\ 1 & \text { if } k>n \text { or } n=0 .\end{cases}
$$

Proof. By (5.1), we have that $\delta_{n}^{\prime}(m)^{k}=\phi_{\mathbf{C}}\left(\left(d_{1}-2\right)\right)^{k}=0$ for $k>n$ or $n=0$. Let $\eta$ be the canonical complex line bundle over $C P^{2 n+1}$. Put $\sigma=\pi\left(Z_{2^{m}}, S^{1}\right)^{*} \eta-1 \in \widetilde{K}_{\mathbf{C}}\left(L^{2 n+1}\left(2^{m}\right)\right)$. Then we have

$$
\begin{aligned}
\pi\left(Z_{\left.2^{m}, D^{*}\left(2^{m+1}\right)\right)^{!} \delta_{n}^{\prime}(m)^{k}}\right. & =\pi\left(\mathbb{Z}_{2^{m}}, S^{1}\right)^{1} \pi\left(S^{1}, S^{3}\right)^{1}\left(\xi_{n}\left(S^{3}\right)_{\mathbf{C}}-2\right)^{k} \\
& =(\sigma+\bar{\sigma})^{k} \\
& =\sigma^{2 k}+\text { higher terms. }
\end{aligned}
$$

By $[13,1.1]$, we have

$$
\# \sigma^{k}= \begin{cases}2^{m+2 n+1-k} & \text { if } 1 \leqq k \leqq 2 n+1 \\ 1 & \text { if } k>2 n+1 .\end{cases}
$$

Then we know that $\# \delta_{n}^{\prime}(m)^{k}$ is a multiple of $2^{m+2(n-k)+1}$ if $1 \leqq k \leqq n$.

To obtain an upper bound of $\# \delta_{n}^{\prime}(m)^{k}$, we use the complex cobordism theory $U^{*}$.

5.3. (Conner-Floyd [7]). There exists a monomorphism $\tilde{K}_{\mathbf{C}}(X) \rightarrow$ $U^{2}(X)$ for any finite connected $C W$-complex $X$.

Since the tangent bundle $\tau N^{n}(m)$ of $N^{n}(m)$ satisfies the condition 


$$
\tau N^{n}(m) \oplus 1 \cong(n+1) \xi_{n}\left(D^{*}\left(2^{m+1}\right)\right)_{\mathbf{R}}
$$

$[22,3.3], N^{n}(m)$ is a $U$-manifold. Then there is a duality isomorphism

$$
U^{k}\left(N^{n}(m)\right) \cong U_{4 n+3-k}\left(N^{n}(m)\right)
$$

and in particular we have

$$
U^{2}\left(N^{n}(m)\right) \cong U_{4 n+1}\left(N^{n}(m)\right)
$$

Since $N^{n}(m)$ is the $4 n+3$-skeleton of $B D^{*}\left(2^{m+1}\right)=\bigcup_{n} N^{n}(m)$ [10], we have

$$
U_{4 n+1}\left(N^{n}(m)\right) \cong U_{4 n+1}\left(N^{n+1}(m)\right) \cong \cdots \cong U_{4 n+1}\left(B D^{*}\left(2^{m+1}\right)\right) .
$$

Hence we have a monomorphism

$$
\tilde{K}_{\mathbf{C}}\left(N^{n}(m)\right) \longrightarrow U_{4 n+1}\left(B D^{*}\left(2^{m+1}\right)\right)
$$

Since $H_{*}\left(B D^{*}\left(2^{m+1}\right) ; \mathbb{Z}\right)$ is periodic $([6, \mathrm{XII}])$, the Atiyah-Hirzebruch spectral sequence for $U_{*}\left(B D^{*}\left(2^{m+1}\right)\right)$ collapses $([16])$ and then the Thom map $\mu: U_{*}\left(B D^{*}\left(2^{m+1}\right)\right) \rightarrow H_{*}\left(B D^{*}\left(2^{m+1}\right)\right)$ is epimorphic. Recall $D^{*}\left(2^{m+1}\right)$ $=\left\{x, y ; x^{2^{m-1}}=(y x)^{2}=y^{2}\right\}$. We will identify $\mathbb{Z}_{2^{m}}$ and $\mathbb{Z}_{4}$ with the subgroups of $D^{*}\left(2^{m+1}\right)$ generated by $x$ and $y$ respectively. Let $i_{1}^{\prime}: \mathbb{Z}_{2^{m}}$ $\rightarrow D^{*}\left(2^{m+1}\right)$ and $i_{2}^{\prime}: \mathbb{Z}_{4} \rightarrow D^{*}\left(2^{m+1}\right)$ be those inclusions. And let $i_{1}: B Z_{2^{m}}$ $=L^{\infty}\left(2^{m}\right) \rightarrow N^{\infty}(m)=B D^{*}\left(2^{m+1}\right)$ and $i_{2}: B Z_{4}=L^{\infty}(4) \rightarrow B D^{*}\left(2^{m+1}\right)$ be the induced maps (see §2). And we will write the following inclusions by the same letter $c$ :

$$
\begin{gathered}
N^{k}(m) \subset B D^{*}\left(2^{m+1}\right), L^{2 k}\left(2^{m}\right) \subset L^{\infty}\left(2^{m}\right)=B Z_{2^{m}} \text { and } \\
L^{2 k}(4) \subset L^{\infty}(4)=B Z_{4} .
\end{gathered}
$$

Then $\left\{\mu\left[N^{k}(m), \iota\right], \mu i_{1 *}\left[L^{2 k}\left(2^{m}\right), \iota\right], \mu i_{2^{*}}\left[L^{2 k}(4), \iota\right] ; 0 \leqq k\right\}$ generates $\tilde{H}_{*}$ $\left(B D^{*}\left(2^{m+1}\right) ; \mathbb{Z}\right)$ and then $\left\{\left[N^{k}(m), \iota\right], i_{1^{*}}\left[L^{2 k}\left(2^{m}\right), \iota\right], i_{2^{\star}}\left[L^{2 k}(4), \iota\right] ; 0 \leqq k\right\}$ generates the $U_{*}$-module $\tilde{U}_{*}\left(B D^{*}\left(2^{m+1}\right)\right)$. The orders of these $U_{*}$ module generators have been computed by K. Shibata and Y. Katsube (unpublished) as follows:

5.4

$$
\#\left[N^{k}(m), \iota\right]=2^{m+2 k+1},
$$




$$
\begin{aligned}
& \# i_{1 *}\left[L^{2 h}\left(2^{m}\right), \iota\right]=\left\{\begin{array}{ll}
2 & \text { if } k=0 \\
2^{m+2 k-1} & \text { if } k>0
\end{array}\right. \text {, and } \\
& \# i_{2^{*}}\left[L^{2 k}(4), \iota\right]=2^{2 k+1} .
\end{aligned}
$$

This implies

$$
2^{m+2 n-1} U_{4 n+1}\left(B D^{*}\left(2^{m+1}\right)\right)=0
$$

and then

$$
2^{m+2 n-1} \widetilde{K}_{\mathbf{C}}\left(N^{n}(m)\right)=0
$$

Comparing this upper bound with the above lower bound of $\# \delta_{n}^{\prime}(m)$, we have

$$
\text { 5.5. } \# \delta_{n}^{\prime}(m)= \begin{cases}2^{m+2 n-1} & \text { if } n>0 \\ 1 & \text { if } n=0 .\end{cases}
$$

To compute $\# \delta_{n}^{\prime}(m)^{k}$, we prepare the following lemma.

Lemma 5.6. (cf. $[19,5.2])$ For $\lambda \in \mathbb{Z}, \alpha \in R_{\mathbf{C}}\left(D^{*}\left(2^{m+1}\right)\right)$ and $k \geqq 2$, $\lambda\left(d_{1}-2\right)=\alpha\left(d_{1}-2\right)^{n+1}$ holds if and only if $\lambda\left(d_{1}-2\right)^{k}=\alpha\left(d_{1}-2\right)^{n+k}$.

Proof. Only if part is trivial. (5.1) and (5.5) imply

$$
2^{m+2 n-1}\left(d_{1}-2\right)=\beta\left(d_{1}-2\right)^{n+1} \quad \text { for some } \beta \in R_{\mathbf{C}}\left(D^{*}\left(2^{m+1}\right)\right),
$$

and in particular

$$
2^{m+1}\left(d_{1}-2\right)=\beta\left(d_{1}-2\right)^{2} \quad \text { for some } \beta \in R_{\mathbb{C}}\left(D^{*}\left(2^{m+1}\right)\right) .
$$

Then

$$
\beta^{k-1}\left(d_{1}-2\right)^{k}=2^{k(m+1)}\left(d_{1}-2\right)
$$

and hence

$$
\beta^{k-1}\left(d_{1}-2\right)^{n+k}=2^{k(m+1)}\left(d_{1}-2\right)^{n+1} .
$$

Let $\lambda\left(d_{1}-2\right)^{k}=\alpha\left(d_{1}-2\right)^{n+k}$. Then 


$$
\begin{aligned}
& 2^{k(m+1)} \lambda\left(d_{1}-2\right)=\lambda \beta^{k-1}\left(d_{1}-2\right)^{k}=\beta^{k-1} \alpha\left(d_{1}-2\right)^{n+k} \\
& =2^{k(m+1)} \alpha\left(d_{1}-2\right)^{n+1} .
\end{aligned}
$$

But $R_{\mathbb{C}}\left(D^{*}\left(2^{m+1}\right)\right)$ is free, so we have

$$
\lambda\left(d_{1}-2\right)=\alpha\left(d_{1}-2\right)^{n+1} .
$$

Thus the lemma (5.6) follows.

(5.6) implies

$$
\sharp \delta_{n}^{\prime}(m)^{k}=\# \delta_{n-k+1}^{\prime}(m) \quad \text { for } \quad 1 \leqq k \leqq n
$$

and hence

$$
\# \delta_{n}^{\prime}(m)^{k}=2^{m+2(n-k)+1} \quad \text { for } \quad 1 \leqq k \leqq n \text {. }
$$

This completes the proof of the proposition.

Since $d_{1}$ is self conjugate (i.e. $t\left(d_{1}\right)=d_{1}$ ), we have

$$
t\left(\delta_{n}^{\prime}(m)\right)=\delta_{n}^{\prime}(m) \quad \text { and } \quad c\left(\delta_{n}(m)\right)=2 \delta_{n}(m)
$$

Then we have

$$
\# \delta_{n}(m)=2^{m+2 n-2} \quad \text { or } \quad 2^{m+2 n-1} \text { 。 }
$$

Pitt $[19,5.5]$ has proved

$$
\# \delta_{1}(m)=2^{m+1} \text {. }
$$

Using (4.7) and the method of Pitt, the author has checked the following proposition.

\section{Proposition 5.7.}

$$
\# \delta_{n}(2)=\left\{\begin{array}{ll}
2^{2 n+1} & \text { if } n \text { is odd } \\
2^{2 n} & \text { if } n \text { is even }
\end{array}, \# \delta_{n}(3)=\left\{\begin{array}{cl}
2^{2 n+2} & \text { if } n \text { is odd } \\
2^{2 n+1} & \text { if } n \text { is positive } \\
\text { and even } \\
1 \quad \text { if } n=0
\end{array}\right.\right.
$$

and 


$$
\# \delta_{2}(m)=2^{m+2} \text {. }
$$

In case $m=2$, this has been proved by Fujii [11], more generally he has determined the additive structure of $K_{\mathbf{R}}\left(N^{n}(2)\right)$. The proof of (5.7) is long and routine and so we omit it.

Remark. By (5.7), we may conjecture that for $n>0$

$$
\# \delta_{n}(m)= \begin{cases}2^{m+2 n-1} & \text { if } n \text { is odd } \\ 2^{m+2 n-2} & \text { if } n \text { is even. }\end{cases}
$$

\section{6. $\bar{J}\left(\mathbb{N}^{n}(2)\right)$}

The purpose of this section is to prove the following theorem.

Theorem 6.1. J-homomorphism $J: \widetilde{K}_{\mathbf{R}}\left(N^{n}(2)\right) \rightarrow J\left(N^{n}(2)\right)$ is an isomorphism.

Since $\Psi_{\mathbf{C}}^{-1}=t$ is identity on $K_{\mathbf{C}}\left(N^{n}(m)\right)$ by (4.2) and (5.1), we have $\Psi_{F}^{k}=\Psi_{F}^{-k}$ on $K_{F}\left(N^{n}(m)\right)$. So we will consider $\Psi_{F}^{k}$ for $k$ non negative only.

Consider Adams operation $\Psi_{F}^{h}: R_{F}(G) \rightarrow R_{F}(G)$. Concerning the characters it is well known that

$$
\chi_{\Psi_{F}^{h}(\theta)}(g)=\chi_{\theta}\left(g^{h}\right) \text { for } \theta \in R_{F}(G) \text { and } g \in G
$$

(see $[1,4.4]$ ). Then (4.2) and a short character computation show that

$$
\Psi_{F}^{k}=\text { identity on } R_{F}\left(D^{*}(8)\right) \text { for } k \text { odd. }
$$

Then (5.1) and the following commutative diagram

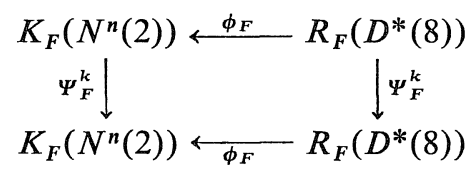

imply

Lemma 6.2. $\Psi_{F}^{k}=$ identity on $K_{F}\left(N^{n}(2)\right)$ for $k$ odd. 
Now we prove Theorem 6.1. Since $\tilde{K}_{F}\left(N_{n}(m)\right)$ is a 2-primary group (see e.g. [6], [10], [11]), we have $2^{N} \widetilde{K}_{\mathbf{R}}\left(N^{n}(2)\right)=0$ for some $N$. Let us choose $e: \mathbb{Z} \rightarrow\{0,1,2, \ldots\}$ so that $e(k) \geqq N$ for $k$ even. Then $k^{e(k)}\left(\Psi_{\mathbb{R}}^{k}\right.$ $-1) K_{\mathbf{R}}\left(N^{n}(2)\right)=0$ for $k$ even. But for $k$ odd $\Psi_{\mathbf{R}}^{k}$ is identity on $K_{\mathbf{R}}\left(N^{n}(2)\right)$ by (6.2), so that $k^{e(k)}\left(\Psi_{\mathbf{R}}^{k}-1\right) K_{\mathbf{R}}\left(N^{n}(2)\right)=0$. Thus we have $Y_{e}=0$ for this function $e$, and hence $\cap Y_{e}=0$ (see $\S 3$ for the definition of $Y_{e}$ ). This completes the proof of Theorem 6.1.

As a corollary of this theorem and (5.7), we have the following.

\section{Corollary 6.3.}

$$
\# J\left(\delta_{n}(2)\right)= \begin{cases}2^{2 n+1} & \text { if } n \text { is odd } \\ 2^{2 n} & \text { if } n \text { is even. }\end{cases}
$$

\section{7. $\mathscr{J}\left(\mathbb{N}^{n}(G)\right)$}

In this section we evaluate the $J$-order of $\xi_{n}(G)$.

For simplicity we will use the notation $J(\tau)$ instead of $J\left(\tau-\operatorname{dim}_{\mathbf{R}} \tau\right)$ for a vector bundle $\tau$.

Consider the induced homomorphism $\pi\left(G, S^{3}\right)^{*}: J\left(H P^{n}\right) \rightarrow J\left(N^{n}(G)\right)$. Then, since $\pi\left(G, S^{3}\right)^{*} J\left(\xi_{n}\left(S^{3}\right)\right)=J\left(\xi_{n}(G)\right)$, we have

Proposition 7.1. \#J( $\left.\zeta_{n}(G)\right)$ is a factor of $B_{n}$.

By (5.2) and (5.7) we have

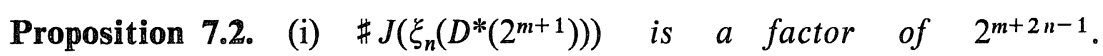

(ii) $\# J\left(\xi_{n}\left(D^{*}(16)\right)\right.$ ) is a factor of $2^{2 n+2}$ (if $n$ is odd) or $2^{2 n+1}$ (if $n$ is even).

(iii) $\# J\left(\xi_{2}\left(D^{*}\left(2^{m+1}\right)\right)\right.$ ) is a factor of $2^{m+2}$.

Let $\mathbb{Z}_{k}$ be a cyclic subgroup of $G$ and $\eta_{2 n+1}(k)$ be the canonical complex line bundle over $L^{2 n+1}(k)$. Since $\pi\left(\mathbb{Z}_{k}, G\right)^{*} \xi_{n}(G)_{\mathbf{C}}=\eta_{2 n+1}(k)$ $+\bar{\eta}_{2 n+1}(k)$, we have

$$
\pi\left(\mathbb{Z}_{k}, G\right)^{*} J\left(\xi_{n}(G)\right)=2 J\left(\eta_{2 n+1}(k)\right)
$$


Then we have

Proposition 7.3. If $Z_{k} \subset G$, then $\# J\left(\xi_{n}(G)\right)$ is a multiple of $\# 2 J\left(\eta_{2 n+1}(k)\right)$.

Remark. \#J( $\left.\eta_{n}(k)\right)$ has been determined by Kambe-Matsunaga-Toda [12] and Kobayashi-Sugawara [15] when $k=p$ or $p^{2}$ for $p$ prime.

When $D^{*}(8) \subset G$ i.e. $G=D^{*}(8 m), T^{*}, O^{*}$ or $I^{*}$, we obtain the following proposition by $(6.3)$, since $\pi\left(D^{*}(8), G\right)^{*} J\left(\xi_{n}(G)\right)=J\left(\xi_{n}\left(D^{*}(8)\right)\right)$.

Proposition 7.4. If $D^{*}(8) \subset G$, then $\# J\left(\xi_{n}(G)\right)$ is a multiple of $2^{2 n+1}$ (if $n$ is odd) or $2^{2 n}$ (if $n$ is even).

As a corollary of this we have

Corollary 7.5. If $n=2^{u}+2 v+1$ for $\quad \frac{u-3}{4} \leqq v \leqq 2^{u-1}-1$ and $u \geqq 1$, then $\# J\left(\xi_{n}\left(D^{*}\left(2^{m+1}\right)\right)\right)=2^{2 n+1}$.

Proof. Recall that

$$
v_{2}\left(B_{n}\right)=\max \left\{2 n+1,2 j+v_{2}(j) ; 1 \leqq j \leqq n\right\}
$$

(see [21]), where $v_{2}(w)$ denotes the largest integer for which $2^{v_{2}(w)}$ divides $w$. If $n$ satisfies the above condition, then $v_{2}\left(B_{n}\right)=2 n+1$ and then (7.1) and (7.4) imply (7.5).

\section{S-types of $\mathbb{N}_{k}^{n+k}(G)$}

Evaluating the (co)homology groups of $N_{k}^{n+k}(G)$ (see [6, XII $\S \S$, $8,9]$ ), we have

Theorem 8.1. If $N_{j}^{m+j}(G)$ and $N_{k}^{n+k}(H)$ are of the same stable homotopy type, then $G$ is conjugate with $H$ and $m=n$.

By Atiyah $[3,2.6]$ and (2.1) we have

Proposition 8.2. If $j \equiv k\left(\# J\left(\xi_{n}(G)\right)\right)$, then $N_{j}^{n+j}(G)$ and $N_{k}^{n+k}(G)$ are of the same stable homotopy typc. 
Put $B_{n}(m)=\min \left\{m+2 n-1, v_{2}\left(B_{n}\right)\right\}$. Then (8.2) implies the following theorem by (5.1), (7.1) and (7.2).

Theorem 8.3. (i) If $j \equiv k\left(2^{B_{n}(m)}\right)$, then $N_{j}^{n+j}(m)$ is $S$-equivalent to $N_{k}^{n+k}(m)$.

(ii) For a fixed $G$, all $N_{n}^{n}(G)$ are of the same stable homotopy type.

(iii) If $m=2$ or 3 and $j \equiv k \bmod \left\{\begin{array}{ll}2^{2 n+m-1} & \text { if } n \text { is odd } \\ 2^{2 n+m-2} & \text { if } n \text { is even }\end{array}\right.$, then $N_{j}^{n+j}(m)$ is S-equivalent to $N_{k}^{n+k}(m)$.

For the converse of this, we have the following theorem by methods of Kobayashi-Sugawara $[15,1.1]$.

Theorem 8.4. If $N_{j}^{n+j}(2)$ and $N_{k}^{n+k}(2)$ are of the same stable homotopy type for $n \geqq 1$, then $j \equiv k\left(2^{2 n-2}\right)$.

Proof. Consider the Puppe exact sequence

$$
\begin{array}{r}
\tilde{K}_{\mathbf{C}}\left(S^{1} \wedge N^{n+j}(m)\right) \stackrel{\left(1 \wedge^{\prime}\right)^{1}}{\longrightarrow} \tilde{K}_{\mathbf{C}}\left(S^{1} \wedge N^{j-1}(m)\right) \\
\stackrel{p^{1}}{\longrightarrow} \tilde{K}_{\mathbb{C}}\left(N^{n+j}(m)\right)
\end{array}
$$

Since Atiyah-Hirzebruch spectral sequences for $\widetilde{K}_{\mathbb{C}}\left(S^{1} \wedge N^{u}(m)\right)$ and $\tilde{K}_{\mathbf{C}}\left(N_{j}^{n+j}(m)\right)$ collapse, we have $\tilde{K}_{\mathbf{G}}\left(S^{1} \wedge N^{u}(m)\right)=\mathbb{Z}$ and $(1 \wedge \iota)^{\prime}=0$. Hence above sequence induces the following exact one

$$
0 \longrightarrow \mathbb{Z} \longrightarrow \tilde{K}_{\mathbf{C}}\left(N_{j}^{n+j}(m)\right) \stackrel{p^{1}}{\longrightarrow} \tilde{K}_{\mathbb{G}}\left(N^{n+j}(m)\right)
$$

and then $p^{1}$ is monomorphic on $\operatorname{Tor}\left(\widetilde{K}_{\mathbf{c}}\left(N_{j}^{n+j}(m)\right)\right)$. Then by (6.2), we know that $\Psi_{\mathbb{G}}^{v}$ is identity on $\operatorname{Tor}\left(\widetilde{K}_{\mathbb{C}}\left(N_{j}^{n+j}(2)\right)\right)$ for $v$ odd. Consider the following diagram

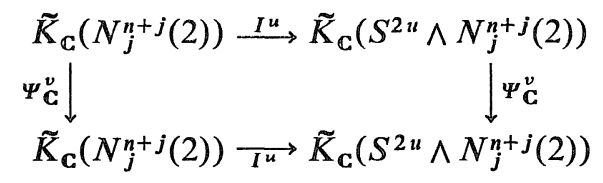

where $I$ indicates the Bott isomorphism. Then we have $\Psi_{\mathbb{C}}^{v} I^{u}=v^{u} I^{u} \Psi_{\mathbb{C}}^{v}$. 
Therefore we have

8.5. $\quad \Psi_{\mathbf{C}}^{2 v+1}=(2 v+1)^{u} \quad$ on $\operatorname{Tor}\left(\tilde{K}_{\mathbf{C}}\left(S^{2 u} \wedge N_{j}^{n+j}(2)\right)\right.$.

If $S^{u} \wedge N_{j}^{n+j}(m)$ is homotopy equivalent to $S^{v} \wedge N_{k}^{n+k}(m)$, then $v=u$ $+4(j-k)$ by their cohomology groups.

Now suppose that there exists a homotopy equivalence

$$
g: s^{2 u+4(j-k)} \wedge N_{k}^{n+k}(2) \longrightarrow S^{2 u} \wedge N_{j}^{n+j}(2)
$$

and consider the following commutative diagram

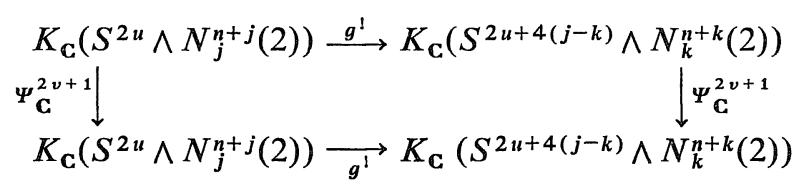

Then (8.5) implies that

8.6. $\quad(2 v+1)^{u+2(j-k)} g^{1}=(2 v+1)^{u} g^{1} \quad$ on $\quad \operatorname{Tor}\left(\tilde{K}_{\mathrm{C}}\left(S^{2 u} \wedge N_{j}^{n+j}(2)\right)\right)$.

Since

$$
\begin{aligned}
\tilde{K}_{\mathbf{C}}\left(S^{2 u} \wedge N_{j}^{n+j}(2)\right) & \cong \tilde{K}_{\mathbf{C}}\left(N_{j}^{n+j}(2)\right) \\
& \cong K_{\mathbf{C}}^{-4 j}\left(N^{n}(2)\right) \quad(\text { Thom isomorphism }) \\
& \cong K_{\mathbf{C}}\left(N^{n}(2)\right)
\end{aligned}
$$

there is an element of order $2^{2 n+1}$ in $\tilde{K}_{\mathbf{C}}\left(S^{2 u} \wedge N_{j}^{n+j}(2)\right)$ by (5.2). Then (8.6) implies that

$$
(2 v+1)^{u+2(j-k)}-(2 v+1)^{u} \equiv 0\left(2^{2 n+1}\right),
$$

that is

$$
(2 v+1)^{2(j-k)}-1 \equiv 0\left(2^{2 n+1}\right) .
$$

It was proved by Adams $[1,8.1]$ that

$$
\text { if } b=(2 a+1) 2^{f}, \text { then } 3^{b}-1 \equiv 2^{f+2}\left(2^{f+3}\right) 。
$$

This implies 


$$
3^{2(j-k)}-1 \equiv 2^{v_{2}(2(j-k))+2}\left(2^{v_{2}(2(j-k))+3}\right) .
$$

Then

$$
2 n+1 \leqq v_{2}(2(j-k))+2=v_{2}(j-k)+3,
$$

and therefore

$$
j-k \equiv 0\left(2^{2 n-2}\right)
$$

This completes the proof of Theorem 8.4.

\section{References}

[1] Adams, J. F., Vector fields on spheres, Ann. of Math. 75 (1962), 603-632.

[2] $\longrightarrow$, On the groups $J(X)-I$, Topology 2 (1963), 181-195.

[3] Atiyah, M. F., Thom complexes, Proc. London Math. Soc. 11 (1961), 291-310.

[4] ${ }^{-}$and Segal, G., Equivariant $K$-theory and completions, J. Differential Geometry 3 (1968), 1-18.

[5] Bredon, G. E., Introduction to compact transformation groups, Academic Press, New York, 1972.

[6] Cartan, H. and Eilenberg, S., Homological Algebra, Princeton Univ. Press, Princeton, New Jersey, 1956.

[7] Conner, P. E., and Floyd, E. E., The relation of cobordism ot $K$-theoreis, Lecture Notes in Math. 28, Springer-Verlag, 1966.

[8] Feder, S. and Gitler, S., Stunted projective spaces and the $J$-order of the Hopf bundle, Bull. Amer. Math. Soc. 80 (1974), 748-749.

[9] - The classification of stunted projective spaces by stable homotopy type, preprint.

[10] Fujii, K., On the $K$-ring of $S^{4 n+3} / H_{m}$, Hiroshima Math. J. 3 (1973), 251265.

[11] - On the KO-rings of $S^{4 n+3} / H_{m}$, Hiroshima Math. J. 4 (1974), 459475.

[12] Kambe, T., Matsunaga, H. and Toda, H., A note on stunted space, J. Math. Kyoto Univ. 5 (1966), 143-149.

[13] Kawaguchi, T. and Sugawara, M., $K$ - and $K O$-rings of the lens space $L^{n}\left(p^{2}\right)$ for odd prime $p$, Hiroshima Math. J. $\mathbb{1}$ (1971), 273-286.

[14] Kobayashi, T. and Sugawara, M., $K_{A}$-rings of lens spaces $L^{n}(4)$, Hiroshima Math. J. $\mathbb{1}$ (1971), 253-271.

[15] Kobayashi, T. and Sugawara, M., On stable homotopy types of stunted lens spaces, Hiroshima Math. J. $\mathbb{1}$ (1971), 281-304.

[16] Landweber, P. S., Complex bordism of Classifying spaces, Proc. Amer. Math. Soc. 27 (1971), 175-179.

[17] Montgomery, D. and Zippin, L., Topological transformation group, Interscience Publishers, Inc., New York, 1955.

[18] Orlik, P., Seifert manifolds, Lecture Notes in Math. 291, Springer (1972). 
[19] Pitt, D., Free actions of generalized quaternion groups on spheres, Proc. London Math. Soc. 26 (1973), 1-18.

[20] Sanderson, B. J., Immersions and embeddings of projective spaces, Proc. London Math. Soc. 14 (1964), 137-153.

[21] Sigrist, F. and Suter, U., Cross-sections of symplectic Stiefel manifolds, Trans. Amer. Math. Soc. 184 (1973), 247-259.

[22] Szczarba, R. H., On tangent bundles of fibre spaces and quotient spaces, Amer. J. Math. 86 (1964), 685-697.

[23] Wolf, J. A., Spaces of constant curvature, McGraw Hill, New York, 1967. 
